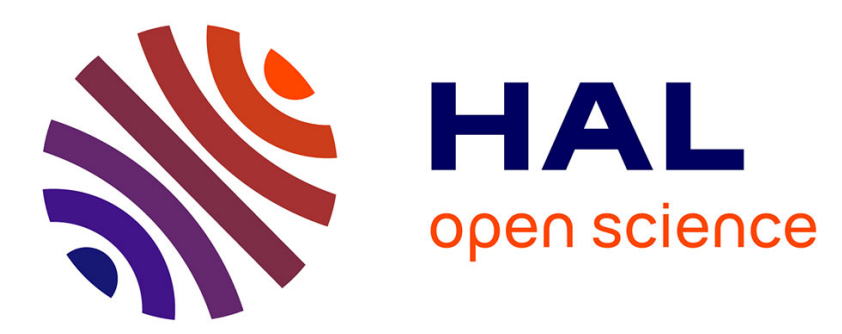

\title{
On the validity of the self-consistent scale transition model for inclusions with varying morphologies
}

Emmanuel Lacoste, Sylvain Fréour, Frédéric Jacquemin

\section{To cite this version:}

Emmanuel Lacoste, Sylvain Fréour, Frédéric Jacquemin. On the validity of the self-consistent scale transition model for inclusions with varying morphologies. Mechanics of Materials, 2010, 42, pp.218 226. 10.1016/j.mechmat.2009.10.002 . hal-01471147

\section{HAL Id: hal-01471147 \\ https://hal.science/hal-01471147}

Submitted on 19 Feb 2017

HAL is a multi-disciplinary open access archive for the deposit and dissemination of scientific research documents, whether they are published or not. The documents may come from teaching and research institutions in France or abroad, or from public or private research centers.
L'archive ouverte pluridisciplinaire HAL, est destinée au dépôt et à la diffusion de documents scientifiques de niveau recherche, publiés ou non, émanant des établissements d'enseignement et de recherche français ou étrangers, des laboratoires publics ou privés. 


\title{
On the validity of the self-consistent scale transition model for inclusions with varying morphologies
}

\author{
E. Lacoste, S. Fréour, F. Jacquemin
}

Institut de Recherche en Génie Civil et Mécanique (UMR CNRS 6183), Université de Nantes - Centrale Nantes, 37 Boulevard de l'Université, BP 406,44602

Saint-Nazaire cedex, France

Scale transition models based on Eshelby's solution provide interesting information on the properties and multi-scale mechanical states experienced by materials presenting complex microstructures, such as composite materials, accounting for the constituents' properties but also microstructural parameters such as the morphology of the heterogeneous inclusions constituting the material. Nevertheless, until now, these approaches cannot reliably account for multiple inclusion morphologies in the same representative elementary volume of the modeled material, as they predict two distinct sets of properties depending of the quantities (strains or stresses) used to formulate the homogenization procedure.

The present work aims to investigate the validity of Kröner-Eshelby self-consistent model for thermo-elastic behaviour, in the case when several morphologies do coexist within the same representative elementary volume. A study of the two resulting formulations and their limits leads to suggest a mixed formulation inspired of Vook-Witt's model, as an understandable but acceptable compromise between the two alternatives. The results of this formulation are also described in the case of a thermo-mechanical load.

Keywords: Kröner-Eshelby self-consistent model Multiple inclusions, Random orientation, Multiscale behaviour, Composite materials

\section{Introduction}

The recent development of composite materials during the last two decades opened new prospects to mechanical part engineering, particularly for aeronautical applications, because of their high strength-to-weight ratio as well as corrosion and fatigue resistance. But, as a counterpart of these advantages, the inherent heterogeneity of these material induce complex mechanical behaviours under service loads, and also internal stresses produced during the cure and tooling processes of a part.

Thus, the need for predicting the stress distribution among the constituents gave a boost to the development of the so-called "scale transition models", which permit to relate the behaviour of the material at several scales of interest. Among these, Eshelby's solution based models (like Mori-Tanaka or Kröner-Eshelby estimates, the latter often referred to as the self-consistent model of the polycrystal) suggest a realistic and interesting approach, which enables to calculate the homogenized properties of the material, and also to predict how the stresses do concentrate between the constituents as a function of the considered load. Eshelby's equations were first used by Kröner to estimate the elastic moduli (Kröner, 1958) and the plastic behaviour (Kröner, 1961) of polycrystals, from the properties of its constitutive crystallites. This elastoplastic model inspired similar studies on the thermo-elastic behaviour of heterogeneous materials (Hutchinson, 1970), then on the time-dependent creep and relaxation of polycrystals (Weng, 1993). These models are more and more used as computation capacities knew a boom in the last decade (François, 1991; Fréour, 2003). They were also found interesting for composite materials, owing to the strong heterogeneity of their constituents. Thereby, several recent papers have shown the relevance of these approaches for 
describing the hygro-mechanical and thermo-mechanical behaviour of composite structures (Fréour et al., 2005, 2006; Jacquemin et al., 2005).

These models have also been applied to new industrial materials that present a microstructure containing inclusions of various morphologies or geometrical orientations (Baptiste, 2003; Le Pen and Baptiste, 2002). This kind of microstructure also corresponds to nano-composites, constituted of very rigid and stretched (about $50 \mathrm{~nm}$-long and 5 nm-wide) carbon nanotubes with random geometrical orientation, embedded in a weak-stiffness organic matrix. Nanotubes are still used at a very reduced volume content (below 1\%), but show extremely interesting mechanical properties and notably a Young's modulus higher than 1 TPa (Treacy et al., 1996), which promises very important future developments.

Eshelby's solution-based homogenization procedures are often formulated from both Hill's averages relations, equating the set average achieved over the mechanical strains (respectively stresses) of every inclusion constituting the effective medium to the corresponding macroscopic quantity (see relations (3) and (4) below). One can show that, when both of them are satisfied, the model is self-consistent, i.e., leads to the same macroscopic effective properties regardless of the equation used. Benveniste (1987) and several authors shown evidence that both Hill's average relation could simultaneously be fulfilled by the Mori-Tanaka and self-consistent models, but at the cost of some restrictions over the materials microstructure: (i) either the inclusions must have the same Base Volume (BVs), i.e., shape and alignment of the elementary single heterogeneous inclusions accounted for achieving the scale-transition process should be strictly identical, (ii) or the material and inclusions must be isotropic, (iii) or only single or two-phase materials can be reliably modeled. This includes randomly oriented spheroidal particles, penny-shaped particles randomly oriented in a layout, aligned fibers in a composite ply, isotropic aggregates... Analytic expressions of the thermo-elastic macroscopic properties of the effective medium have been elegantly determined for these cases in a series of paper from Benveniste, Dvorak, and Chen (1991, 1992). Some applications also can be found in Pham (2000) or Qiu and Weng (1991).

However, in the general case of "multi-phased" materials (i.e., whose microstructure involves more than two consistuents) with inclusions of several shapes, Benveniste et al. (1991) demonstrated that the Mori-Tanaka and self-consistent approximations led to unsymmetric stiffnesses, and problems related to the simultaneous fulfilling of Hill's average relations. At the present time, the computation of thermo-elastic properties for materials exhibiting a complex microstructure while presenting an anisotropic behaviour at various scales still seems to constitute an open problem. The ambition of this paper is to decline the well-known classical self-consistent scheme in the case of multimorphous and multiphased materials. Hill's averages principles (see beneath) are used to define validity criterions, and to give an enhanced estimate of the macroscopic thermo-elastic properties.

In a first part, the classical scale transition formalism set up by Hill and Eshelby is extended to the case when mate- rials present a complex morphological microstructure (i.e., when the uniqueness of the BV morphology is not satisfied). The formulations based on the volume averages over stresses and strains are described within the framework of a Kröner-Eshelby model with multiple morphologies, leading to two sets of relations for the effective properties.

In a second part, the model is used to describe the multiscale behaviour of a composite material with a random distribution of the geometrical orientation of the reinforcing strips. A two-steps scale transition homogenization procedure is developed and applied to the material; the results for effective properties and respect of Hill's averages principles are discussed for the two formulations. A mixed formulation is then suggested and validated.

In the last part, this formulation is used to predict local stresses in the material subjected to mechanical and thermal loadings.

\section{General presentation of the multimorphous model}

\subsection{Hill's formalism and Kröner-Eshelby model}

The Kröner-Eshelby scale transition model (more commonly called "self-consistent model") is based on a representation of the material at several scales: on one hand, the "local" scale denoted by the superscript ${ }^{\mathrm{i}}$, where one observes the behaviour of each constituent, considered as an ellipsoidal and homogeneous inclusion (also called Base Volume or BV); on the other hand, the macroscopic scale denoted by the superscript I, where one can observe the behaviour of the Effective Medium (or EM). As thermoelastic behaviours are considered here, they are expressed by the following laws:

$\sigma^{I}=L^{I}:\left(\varepsilon^{I}-\alpha^{I} \Delta T\right)$

$\sigma^{i}=L^{i}:\left(\varepsilon^{i}-\alpha^{i} \Delta T\right)$

In these relations, the stiffnesses are represented by the 4 th-order tensors $L$, and the Coefficients of Thermal Expansion (CTE) by the 2nd-order tensors $\alpha$. The temperature increment is denoted by $\Delta T$, whereas $\sigma$ and $\varepsilon$ stand for the stress and strain, respectively.

The scale transition relations are basically written as volume averages operations on stresses and strains. Hill (1967) showed, in a very general way, the equivalence between set (i.e., volume) averages and volume integrals. Hill's volume average relations over the mechanical states (also called consistency principles on mechanical states) are written:

$\varepsilon^{I}=\left\langle\varepsilon^{i}\right\rangle$

$\sigma^{I}=\left\langle\sigma^{i}\right\rangle$

where the square brackets $\langle\ldots\rangle$ represent the arithmetical volume average.

In a fundamental work, Eshelby studied the behaviour of an inclusion embedded in a homogeneous medium loaded at the infinite (Eshelby, 1957). He demonstrated that, if and only if the inclusion had an ellipsoidal shape, the local stresses and strains where homogeneous inside the BV. Hill (1965) was the first to write the following 
relation between local and overall states, where $L^{*}$ is widely known as Hill's constraint tensor:

$\sigma^{i}-\sigma^{I}=-L^{*}:\left(\varepsilon^{i}-\varepsilon^{I}\right)$

This scheme is often called the "dilute approximation" as it neglects any inter-particles interactions. The self-consistent and Mori-Tanaka models both use this relation, considering the constituents as Eshelby's inclusions. In the mori-Tanaka scheme, one of the constituents (often called the "matrix phase") is considered as the embedding medium; the model is mainly adapted for materials whose matrix phase volume fraction dominates all the others. On the contrary, within the self-consistent model, the embedding medium is given the properties of the EM. As a consequence, the expressions giving the effective properties are implicit and require iterative solving methods, which only give numerical solutions. However, this approach is better adapted than Mori-Tanaka's for materials where all the phases have comparable volume fractions, as inter-particle interactions are indirectly taken in count via the EM (Berryman and Berge, 1996).

Hill's constraint tensor can be written from the Eshelby tensor $S_{e s h}^{I}$ or the Morris tensor $E^{I}$ (also called "influence tensor" $P$ ), thanks to the following relation (where $I$ is the 4 th-order Identity tensor):

$L^{*}=L^{I}:\left(S_{e s h}^{I}{ }^{-1}-I\right)=\left(E^{I^{-1}}-L^{I}\right)$

The Morris tensor expresses the interaction of an inclusion with a given morphology, independently of its elastic properties, and the EM (Morris, 1970). In the case of an ellipsoidal inclusion whose principal axes lengths are $\left\{2 \mathrm{a}_{1}, 2 \mathrm{a}_{2}\right.$, $\left.2 a_{3}\right\}$, it is written in the coordinate system of the inclusion:

$E_{i j k l}^{I}=\frac{1}{4 \pi} \int_{0}^{\pi} \sin \theta \cdot d \theta \int_{0}^{2 \pi} \gamma_{i j k l}^{I} \cdot d \varphi$ with $\gamma_{i j k l}^{I}=\left(K_{i k}^{I}\right)^{-1} \cdot \xi_{j} \cdot \xi_{l}$

In the case of an orthotropic macroscopic symmetry, the $K_{i k}(\xi)$ components were given by Kröner (1953):

$K^{I}=\left[\begin{array}{ccc}L_{11}^{I} \xi_{1}^{2}+L_{66}^{I} \xi_{2}^{2}+L_{55}^{I} \xi_{3}^{2} & \left(L_{12}^{I}+L_{66}^{I}\right) \xi_{1} \xi_{2} & \left(L_{13}^{I}+L_{55}^{I}\right) \xi_{1} \xi_{2} \\ \left(L_{12}^{I}+L_{66}^{I}\right) \xi_{1} \xi_{2} & L_{66}^{I} \xi_{1}^{2}+L_{22}^{I} \xi_{2}^{2}+L_{44}^{I} \xi_{3}^{2} & \left(L_{23}^{I}+L_{44}^{I}\right) \xi_{2} \xi_{3} \\ \left(L_{13}^{I}+L_{55}^{I}\right) \xi_{1} \xi_{2} & \left(L_{23}^{I}+L_{44}^{I}\right) \xi_{2} \xi_{3} & L_{55}^{I} \xi_{1}^{2}+L_{44}^{I} \xi_{2}^{2}+L_{33}^{I} \xi_{3}^{2}\end{array}\right]$

where $\xi_{1}=\frac{\sin \theta \cos \varphi}{a_{1}}, \xi_{2}=\frac{\sin \theta \sin \varphi}{a_{2}}, \xi_{3}=\frac{\cos \theta}{a_{3}}$.

Some computations of the Morris tensor are given in (Kocks et al., 1998; Mura, 1982), who also give a detailed presentation of the Kröner-Eshelby model. Because of the complex expression of the integrand $\gamma_{i j k l}^{l}$, one cannot generally give an analytical expression of $E^{I}$, except for some specific configurations (fibers, discs and spheres in particular). The calculation of this tensor is thereby a key-point of the models based on Eshelby's inclusion.

Furthermore, specific attention must be paid to the fact that the calculation of the Morris tensor must be made with the stiffness tensor $L^{I}$ dropped in the coordinate system $\mathrm{Ri}$ of the inclusion. In the lack of an isotropy with respect to the rotation carried out, several new non-null components appear in $L^{I}$, thus invalidating the expression of $K^{I}$ given by Kröner.
If the inclusions constituting the material do not present a single morphology in the macroscopic coordinate system RI, the tensors $L^{*}, S_{\text {esh }}^{I}$ and $E^{I}$ are not purely macroscopic anymore but related to the considered BV also; in consequence, the superscript ${ }^{\text {I }}$ (or ${ }^{*}$ ) will be replaced by ${ }^{\text {Ii }}$ $\left(\right.$ or $\left.^{*} i\right)$. The transition between the local and macroscopic coordinate systems is made through the convention introduced by Roe (1965). In order to lighten the equations, the tensors will be implicitly dropped in RI for the averaging operations.

\subsection{Formulation with stresses and strains}

Starting form the local and macroscopic behaviour laws, and using the scale transition relation given above (Eq. (7)), one can express the local stresses and strains as:

$$
{ }_{R i} \varepsilon^{i}={ }_{R i}\left(L^{i}+L^{* i}\right)^{-1}:\left[{ }_{R i}\left(L^{I}+L^{* i}\right):{ }_{R i} \varepsilon^{I}+{ }_{R i}\left(L^{i}: \alpha^{i}-L^{I}: \alpha^{I}\right) \Delta T\right]
$$

$$
\begin{aligned}
{ }_{R i} \sigma^{i}= & { }_{R i} L^{i}:{ }_{R i}\left(L^{i}+L^{* i}\right)^{-1}:\left\lfloor_{R i}\left(L^{I}+L^{* i}\right):{ }_{R i} L^{I^{-1}}:{ }_{R i} \sigma^{I}\right. \\
& \left.+{ }_{R i} L^{* i}:{ }_{R i}\left(\alpha^{I}-\alpha^{i}\right) \Delta T\right\rfloor
\end{aligned}
$$

One would notice that the term ${ }_{R i}\left(L^{i}+L^{* i}\right)^{-1}:_{R i}\left(L^{I}+L^{* i}\right)$ which appears in (8) is equivalent to the elastic strain localization tensor: ${ }_{R i} A_{i}^{i}=\left[{ }_{R i} E^{I i}:\left({ }_{R i} L^{i}-{ }_{R i} L^{I}\right)+I\right]^{-1}$. The same is true for the elastic stress concentration tensor: $B^{i}=L^{i}: A^{i}: L^{I^{-1}}$ that appears in the expression of the local stresses. These tensors are defined, for elastic loads, by the two relations:

$\varepsilon^{i}=A^{i}: \varepsilon^{I}$
$\sigma^{i}=B^{i}: \sigma^{I}$

Of course, Hill's averages principles imply the following relation on $A^{i}$ and $B^{i}$ :

$$
\left\langle A^{i}\right\rangle=\left\langle B^{i}\right\rangle=I
$$

Hill's averages principles can be written on both strains and stresses; nevertheless, only one relation is needed to obtain the effective properties, which leads to two alternate expressions.Thus, if using Hill's average principle on stress, one would write the macroscopic stress as:

$$
\begin{aligned}
\sigma^{I}= & \left\langle L^{i}:\left(L^{i}+L^{* i}\right)^{-1}:\left(L^{I}+L^{* i}\right): L^{I^{-1}}\right\rangle: \sigma^{I} \\
& +\left\langle L^{i}:\left(L^{i}+L^{* i}\right)^{-1}: L^{* i}:\left(\alpha^{I}-\alpha^{i}\right)\right\rangle \Delta T
\end{aligned}
$$

The relation being satisfied for any macroscopic state $\left\{\sigma^{I}, \Delta\right.$ T\}, one obtains:

$$
\begin{aligned}
& \left\langle L^{i}:\left(L^{i}+L^{* i}\right)^{-1}:\left(L^{I}+L^{* i}\right): L^{I^{-1}}\right\rangle=\left\langle L^{i}: A^{i}: L^{I^{-1}}\right\rangle=\left\langle B^{i}\right\rangle=I \\
& \left\langle L^{i}:\left(L^{i}+L^{* i}\right)^{-1}: L^{* i}:\left(\alpha^{I}-\alpha^{i}\right)\right\rangle=0
\end{aligned}
$$

The relation (14) implies the following expression for the effective stiffness:

$L^{I}=\left\langle L^{i}: A^{i}\right\rangle$

In a same way, a few algebraic manipulations of the relation (15) give an expression of the effective CTE: 
$\alpha^{I}=\left\langle L^{i}:\left(L^{i}+L *^{i}\right)^{-1}: L *^{i}\right\rangle^{-1}:\left\langle L^{i}:\left(L^{i}+L^{* i}\right)^{-1}: L^{* i}: \alpha^{i}\right\rangle$

On the contrary, if one uses Hill's average principle over the strains, one would obtain:

$\varepsilon^{I}=\left\langle\left(L^{i}+L^{* i}\right)^{-1}:\left[\left(L^{I}+L^{* i}\right): \varepsilon^{I}+\left(L^{i}: \alpha^{i}-L^{I}: \alpha^{I}\right) \Delta T\right]\right\rangle$

which implies the two following relations on elastic and thermal strains:

$$
\begin{aligned}
& \left\langle\left(L^{i}+L^{* i}\right)^{-1}:\left(L^{I}+L^{* i}\right)\right\rangle=\left\langle A^{i}\right\rangle=I \\
& \left\langle\left(L^{i}+L^{* i}\right)^{-1}:\left(L^{i}: \alpha^{i}-L^{I}: \alpha^{I}\right)\right\rangle=0
\end{aligned}
$$

One would then obtain the following effective properties:

$L^{I}=L^{I}:\left\langle A^{i}\right\rangle^{-1}=\left\langle L^{i^{-1}}: B^{i}\right\rangle^{-1}$

$\alpha^{I}=L^{I^{-1}}:\left\langle\left(L^{i}+L^{* i}\right)^{-1}\right\rangle^{-1}:\left\langle\left(L^{i}+L^{* i}\right)^{-1}: L^{i}: \alpha^{i}\right\rangle$

Several authors have shown that, if the inclusions had a unique morphology in the macroscopic coordinate system, the two formulations led to a same set of effective properties. That's why the model is widely used for metals (Fréour, 2003) constituted by spherical inclusions, and for unidirectional composite plies with organic (Fréour et al., 2006) or metallic matrix (Le Pen and Baptiste, 2002). On the other hand, in the case that the material exhibits a morphological texture with a distribution of the inclusion geometry, one obtains two solutions that satisfy Hill's average principle on strains or stresses, but never both of them simultaneously. Benveniste (1987) noticed this drawback of the Eshelby-based models, but no systematic study of it can be found in the bibliography. As a consequence, this numerical study will be achieved in the next section of the present work and compared the results given by each formulation, for a composite material with planar isotropy.

\section{Application to an in-plane isotropic material}

The present application is focused on a high-performance composite material developed for the design of composite parts to be used for aeronautical applications. This material is made of unidirectional reinforcing strips with rectangular shape $(60 \times 8 \times 0.15 \mathrm{~mm})$ and randomly disposed in the layout (see Fig. 1 below).
The strips themselves are composed of T300 fibers and epoxy matrix, set as an unidirectional ply. The material then appears as a thick layout $(1.3 \mathrm{~mm})$ with in-plane isotropy. The properties of the epoxy resin and the T300 fibers are given in (Jacquemin et al., 2005; Agbossou and Pastor, 1997); they are summed up in the Table 1 . In order to lighten the expressions, a "local" coordinate system $R_{x y z}$, oriented along the axis of the fibers constituting the strips, is introduced. This coordinate is obtained by a rotation $\Theta$ around the 3-axis of the "global" coordinate system $R_{123}$ bound to the effective material.

\subsection{Description of the scale transition procedure}

The self-consistent model is used in order to perform a two-steps scale transition (see Fig. 2 below): first, the effective properties of the reinforcing strip are estimated from those of the intra-reinforcements matrix and the carbon fibers. Then, a homogenization procedure is achieved in order to find the behaviour of the material, from the properties of the extra-reinforcements matrix and those of the reinforcing strips (previously estimated).

The homogenization of the reinforcing strip is not a particular matter as it corresponds to the case, treated in a recent paper (Jacquemin et al., 2005), of an unidirectionally reinforced composite ply. Consequently, one will only give the effective properties of the reinforcing strip (see Table 1 ), accounting for a fiber volume fraction of $63 \%$.

The second homogenization step is more problematic, as it involves inclusions of various shapes and (possibly strongly) anisotropic constituents and EM. As a consequence its modelling will be achieved through the "multimorphous" model, as described in the next section.

\subsection{Effective properties of the material for each formulation}

The Eqs. (16) and (21) provide the effective stiffness of the material with $10^{-3}$ accuracy, for both formulations. The averaging operations are achieved onto the two previously described constituents (reinforcing strips with 95\% volume ratio and extra-reinforcement matrix with $5 \%$ ratio). A set of 10 orientations uniformly distributed on $180^{\circ}$ in the $1-2$ plane is considered in order to account for the morphological texture. The obtained effective elastic moduli are reported in the Table 2 .

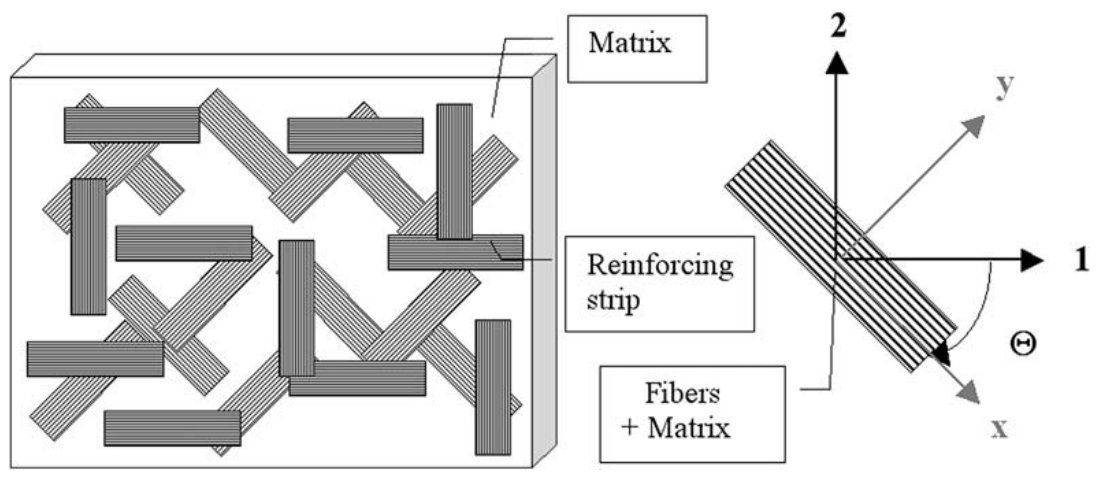

Fig. 1. Schematic representation of the microstructure of the material. 
Table 1

Thermomechanical properties of a reinforcing strip (estimated by the self-consistent model), and its constituents.

\begin{tabular}{|c|c|c|c|c|c|c|c|c|c|}
\hline & \multicolumn{6}{|c|}{ Mechanical moduli } & \multicolumn{2}{|l|}{ CTE } & \multirow{2}{*}{$\begin{array}{l}\text { Density } \\
\rho\left(\mathrm{kg} / \mathrm{m}^{3}\right)\end{array}$} \\
\hline & $E_{x}(\mathrm{GPa})$ & $E_{y}, E_{z}(\mathrm{GPa})$ & $v_{x y}, v_{x z}$ & $G_{x y}, G_{x z}(\mathrm{GPa})$ & $v_{y z}$ & $\mathrm{G}_{\mathrm{yz}}(\mathrm{GPa})$ & $\alpha_{x}\left(10^{-6} / \mathrm{K}\right)$ & $\alpha_{y}, \alpha_{z}\left(10^{-6} / \mathrm{K}\right)$ & \\
\hline Reinforcing strips & 146.8 & 10.2 & 0.274 & 7.0 & 0.355 & 3.8 & -0.620 & 48.0 & 1620 \\
\hline N5208 matrix & 4.5 & 4.5 & 0.4 & 1.61 & 0.4 & 1.61 & 60 & 60 & 1200 \\
\hline
\end{tabular}

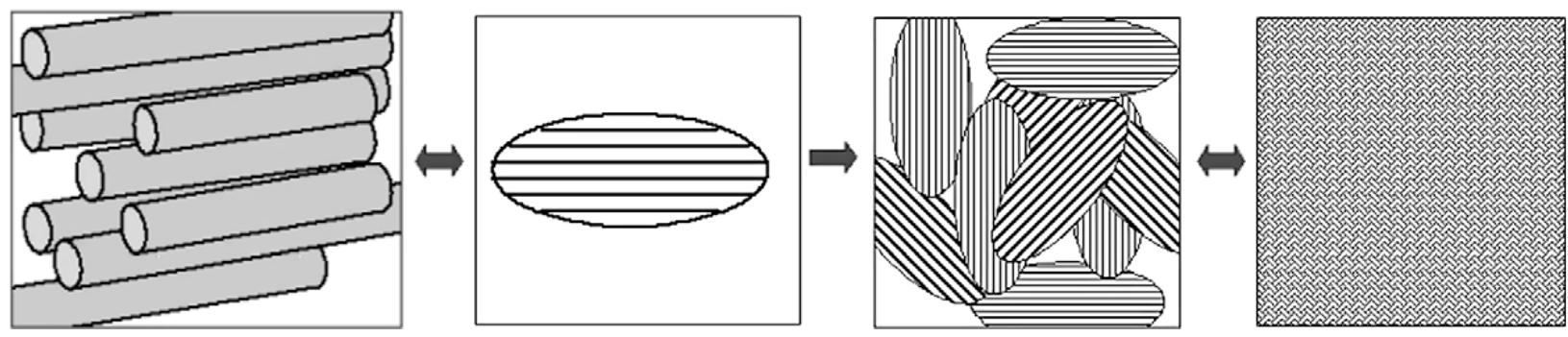

Fig. 2. Schematic representation of the two steps scale transition.

These moduli verify, within the prescribed accuracy, the Reuss and Voigt bounds (see Table 2). The elastic moduli obtained according to the homogenization procedure satisfying Hill's average principle over strains are close to the Reuss bound; whereas those obtained by the approach satisfying Hill's average principle over stresses are practically merged with the Voigt bound.

Furthermore, the two methods lead to drastically different stiffnesses, particularly for the components that govern the in-plane behaviour $\left(E_{1}, v_{12}\right.$ and $\left.G_{12}\right)$. On the contrary, the "out-of-plane" components $\left(E_{3}, v_{13}\right.$ and $\left.G_{13}\right)$ do not vary very much from a homogenization procedure to another.

Also, the computation of the averages for localization and concentration tensors, upon the two constituents and all the orientations, show that none of the two methods simultaneously respects both Hill's averages relations (3) and (4). Actually, according to Table 3, Eq. (16) satisfies
(4) but not (3), whereas Eq. (21) ensures that (3) is satisfied, but not (4) anymore. The numerical error is concentrated on the "in-plane traction-compression" components $\left(A_{11}=A_{22}\right)$, on "in-plane shear" $\left(A_{66}\right)$ and mainly on out-of-plane components $\left(A_{44}=A_{55}\right.$ and $\left.A_{31}=A_{32}\right)$. Nevertheless, it should be noticed that these errors keep relatively weak for the chosen morphology, in the order of $3 \%$ if the out-of-plane shear components are not considered.

On the contrary, for the homogenization with strains, the average relation is insured for strains but not stresses (see Table 3 ). The errors are particularly significant for the in-plane components ( $250 \%$ relative error on $B_{11}=B_{22}$ and $60 \%$ absolute error on $B_{12}=B_{21}$ ), for the in-plane shear (190\% error on $\left.B_{66}\right)$, as well as out-of-plane transverse components $\left(120 \%\right.$ error on $\left.B_{13}=B_{23}\right)$. On the other hand, the error is null for the out-of-plane components of compression-traction $\left(B_{33}\right)$ and shear $\left(B_{44}=B_{55}\right)$.

Table 2

Elastic moduli of the material (isotropic in the 1-2 plan), estimated by the self-consistent model, for the two formulations.

\begin{tabular}{llclrr}
\hline & $E_{1}, E_{2}(\mathrm{GPa})$ & $E_{3}(\mathrm{GPa})$ & $v_{12}$ & $G_{12}(\mathrm{GPa})$ & $G_{13}, G_{23}(\mathrm{GPa})$ \\
\hline$H \varepsilon$ & 16.63 & 9.92 & 0.121 & 7.42 & 0.337 \\
$H \sigma$ & 55.48 & 11.24 & 0.290 & 21.49 & 0.266 \\
Voigt bound & 55.46 & 11.28 & $\mathrm{X}$ & 21.49 & $\mathrm{X}$ \\
Reuss bound & 15.44 & 9.66 & $\mathrm{X}$ & 6.65 & $\mathrm{X}$ \\
\hline
\end{tabular}

Remark: The notations $H \sigma$ and $H \varepsilon$ are used, respectively, to denote the stress-based and strain-based formulations.

Table 3

Averages of the localization and concentration tensors for the in-plane isotropic material, homogenized with stresses or strains.

\begin{tabular}{lllcccc}
\hline$\left\langle A^{i}\right\rangle$ & $A_{11}=A_{22}$ & $A_{33}$ & $A_{44}=A_{55}$ & $A_{66}$ & $A_{12}=A_{21}$ & $A_{13}=A_{23}$ \\
\hline$H \varepsilon$ & 1.001 & 1 & 0.5 & 0.5 & 0 & 0 \\
$H \sigma$ & 1.031 & 1 & 0.443 & 0.512 & 0.007 & 0 \\
$\left\langle B^{i}\right\rangle$ & $B_{11}=B_{22}$ & $B_{33}$ & $B_{44}=B_{55}$ & $B_{66}$ & $B_{12}=B_{21}$ & $B_{13}=B_{23}$ \\
\hline$H \varepsilon$ & 3.465 & 1 & 0.5 & 1.431 & 0.604 & $B_{31}=B_{32}$ \\
$H \sigma$ & 1 & 1 & 0.5 & 0.5 & 0 & 0 \\
Expected value & 1 & 1 & $1 / 2$ & $1 / 2$ & 0 & 0 \\
\hline
\end{tabular}


For both formulations, the in-plane components $(11,12$ and ${ }_{66}$ components) exhibit very important errors over Hill's averages principles. For them, the stress-based formulation gives the weaker errors; on the contrary, for the out-of-plane components, the strain-based formulation is the most reliable. One can also notice that the in-plane moduli $E_{1}, v_{12}$ and $G_{12}$ are the most dependent of the formulation used. Comparatively, the out-of-plane moduli $E_{3}, v_{13}$ and $G_{13}$ are relatively identical whatever the formulation used.

As explained above, the Coefficients of Thermal Expansion have been computed with respect to the two homogenization approaches previously presented in Section 2.2. The thermal homogenization respecting Hill's average relations expressed over the strains is achieved owing to relation (22), using the effective stiffness obtained by relation (21); respectively, the stress-based homogenization is done using the relation (17) and the effective stiffness obtained according to relation (16). This leads to the results presented in the Table 4 below. As for the stiffness, a significant deviation between the results occurs, depending on the homogenization procedure used. By the way, this discrepancy also exists in the case that the same stiffness is used for the two formulations.

In order to quantify the relevance of these results, the adimensionnal errors $X^{\text {thermo }}$ and $Y^{\text {thermo }}$ are defined as follows:

$\left\{\begin{array}{l}X^{\text {thermo }}=\left\langle\Delta \varepsilon^{\text {thermo }, i}\right\rangle \cdot\left(\alpha^{I} \Delta T\right)^{-1} \\ Y^{\text {thermo }}=\left\langle\Delta \sigma^{\text {thermo }, i}\right\rangle \cdot\left(L^{I}: \alpha^{I} \Delta T\right)^{-1}\end{array}\right.$

where $\left\{\begin{array}{l}\Delta \sigma^{\text {thermo }, i}=L^{i}:\left(L^{i}+L^{* i}\right)^{-1}: L^{* i}:\left(\alpha^{I}-\alpha^{i}\right) \Delta T \\ \Delta \varepsilon^{\text {thermo }, i}=\left(L^{i}+L^{* i}\right)^{-1}:\left(L^{i}: \alpha^{i}-L^{I}: \alpha^{I}\right) \Delta T\end{array}\right.$

These errors are also presented in Table 4, for both formulations. One can thus observe that the strain-based formulation verifies Hill's averages principle over strains, but leads to some errors on thermal stresses and mainly in the normal direction. Respectively, the stress-based formulation verifies Hill's averages principle over stresses but underestimates the in-plane thermal strains by more than $200 \%$.

Furthermore, some tests were made to study the evolution of the properties with the morphology of the reinforcing strips. One could observe that the three formulations merged when the inclusions have a circular (or pennyshaped) morphology, thus confirming the validity of the model for a unique morphology. Nevertheless, the gap between the two solutions, as well as the associated errors, quickly grows up as the morphology is stretched and thick.

\subsection{Mixed formulation and results}

From the study carried out in the previous section, one could conclude that none of the two discussed homogenization procedures can give satisfying results for the overall thermo-mechanical behaviour. But one can also point out that each one has a "preferential direction" where exhibited errors are weak: thus, as an example, the stress-based formulation gives a satisfying description of the in-plane behaviour, while the strain-based formulation is well adapted to describe the out-of-plane behaviour.

The microstructure presented by the studied materials corresponds to an assembly of thin in-plane layers. Previous works achieved on similar structures suggested that extreme direction-dependent homogenization procedures such as the Vook-Witt model (see Vook and Witt, 1968; Welzel and Fréour, 2007) gave satisfying results on both in-plane and out-of-plane behaviour. A similar mixed homogenenization is thereby suggested, where the inplane behaviour is modeled using the stress-based formulation, whereas the strain-based formulation is used for the out-of-plane behaviour.

With this mixed formulation, the stiffness tensor of the material satisfies the following from:

$$
L^{I}=\left[\begin{array}{llllll}
{ }^{H \sigma} L_{11}^{I} & { }^{H \sigma} L_{12}^{I} & { }^{H \varepsilon} L_{13}^{I} & & & \\
{ }^{H \sigma} L_{12}^{I} & { }^{H \sigma} L_{22}^{I} & { }^{H \varepsilon} L_{23}^{I} & & & \\
{ }^{H \varepsilon} L_{31}^{I} & { }^{H \varepsilon} L_{32}^{I} & { }^{H \varepsilon} L_{33}^{I} & & & \\
& & & { }^{H \varepsilon} L_{44}^{I} & & \\
& & & & { }^{H \varepsilon} L_{55}^{I} & \\
& & & & & \\
& & & & & { }^{H \sigma} L_{66}^{I}
\end{array}\right]
$$

By injecting this expression in the computation loop of the stiffness, one obtains the results summed up in Table 5. One finds effective moduli very close to those obtained with the stress-based formulation (see Table 2), exception made of the $G_{13}$ coefficient which is closer to the modulus homogenized with strains. It was noticed very little evolution of these elastic moduli if the shape ratios defining the morphology were moderately modified.

Furthermore, the computation of the averages of the localization and concentration tensors (Table 6) shows that Hill's averages principles are relatively well verified. The main errors are committed on the in-plane strains, for compression-traction ( $3 \%$ error on $A_{11}$ ) or shear strains ( $2 \%$ error on $A_{66}$ ). These errors grow up with the stretching and the thickness of the reinforcing strips, but are much lower than those occurring according to the classical formulations.

Table 4

CTEs of the in-plane isotropic material, estimated by the self-consistent model, for the two formulations, and associated errors.

\begin{tabular}{|c|c|c|c|c|c|c|}
\hline & \multicolumn{2}{|l|}{ CTE } & \multicolumn{2}{|c|}{ Error on $\varepsilon$} & \multicolumn{2}{|c|}{ Error on $\sigma$} \\
\hline & $\alpha_{1}, \alpha_{2}\left(10^{-6} / \mathrm{K}\right)$ & $\alpha_{3}\left(10^{-6} / \mathrm{K}\right)$ & $X_{1}^{\text {thermo }}$ & $X_{3}^{\text {thermo }}$ & $Y_{1}^{\text {thermo }}$ & $Y_{3}^{\text {thermo }}$ \\
\hline$H \varepsilon$ & 24.8 & 49.9 & 0 & 0 & -2.088 & 0 \\
\hline$H \sigma$ & 3.52 & 114.9 & 0.062 & -0.424 & 0 & 0 \\
\hline Expected value & $\sim 4$ & $\sim 60$ & 0 & 0 & 0 & 0 \\
\hline
\end{tabular}


Table 5

Elastic moduli of the in-plane isotropic material, with the mixed formulation.

\begin{tabular}{llllll}
\hline$E_{1}, E_{2}(\mathrm{GPa})$ & $E_{3}(\mathrm{GPa})$ & $v_{12}$ & $G_{12}(\mathrm{GPa})$ & $v_{13}, v_{23}$ & $G_{13}, G_{23}(\mathrm{GPa})$ \\
\hline 55.41 & 11.20 & 0.289 & 21.49 & 0.279 & 4.496 \\
\hline
\end{tabular}

The same method is used for computing the effective CTE, which satisfies the following symmetry (Eq. 26):

$$
\alpha^{I}=\left[\begin{array}{lll}
{ }^{H \sigma} \alpha_{11}^{I} & & \\
& H \sigma \alpha_{22}^{I} & \\
& & { }^{H \varepsilon} \alpha_{33}^{I}
\end{array}\right]
$$

The resulting CTE are given in Table 7, which also sums up the errors on thermal stresses and strains, for the studied morphology $(60 \times 8 \times 0.15)$. The mixed formulation provides good results in the considered case, with an error below $3 \%$ on thermal strains. By the way, this error becomes null for a penny-shaped morphology $\left(a_{1}=a_{2}\right)$.

\section{Application to thermo-mechanical loads}

The proposed scale transition procedure enables to compute effective properties closed to experimental results; furthermore, it has been shown that the procedure enabled to estimate local stresses and strains being consistent with the macroscopic loads. In this last section, this procedure will be used in order to predict the internal stresses in the material when subjected to mechanical and thermal loads.

\subsection{Response of the material to purely mechanical load}

In order to describe the multi-scale mechanical behaviour of the material, a macroscopic tensile load, in the 1direction, of $100 \mathrm{MPa}$ is considered. Relation (9) is used to compute the local strains in the constituents, dropped in the local coordinate system Ri. The stresses into the reinforcing strips and the extra-reinforcements matrix, then into the very constituents of the reinforcing strip (matrix and fibers), are computed.

One can observe (see Fig. 3 below) that compressiontraction stresses $\left\{\sigma_{x x}, \sigma_{y y}, \sigma_{z z}\right\}$ evolve as $\pi$-periodic cosinusoids, while shear stresses $\sigma_{x y}$ evolve as sinusoids. These evolutions with orientation match the ones observed for composite laminates subjected to compression-traction loads. Inside the in-plane isotropic material, the in-plane stresses are strongly heterogeneous (contrarily to the inplane strains, which are rather almost homogeneous): the reinforcing strips experience up to $260 \mathrm{MPa}$ in the $x$ direction, while the organic matrix undergoes less than $10 \mathrm{MPa}$. This concentration of stresses is a result of the orientation mismatch between the reinforcement strips and the solicitations. On the contrary, out-of-plane stresses are rather homogeneous and remains very weak.

The concentration of stresses is increased into the fiber constituting the reinforcement strips, which experience more than $400 \mathrm{MPa}$ while the matrix takes less than $20 \mathrm{MPa}$. One will also notice the emergence of low complementary stresses ( -2 to $2.5 \mathrm{MPa}$ ) inside the fibers and the intra-reinforcement matrix, along the $z$-direction.

\subsection{Response of the material to purely thermal load}

A similar study was achieved for the in-plane isotropic material submitted to a $-100{ }^{\circ} \mathrm{C}$ thermal load. This kind of load is typical of the cooling occurring during the cure process of composite materials, which is reported to possibly induce severe residual stresses (Guemes, 1994; Ogi et al., 1999).

Contrarily to the mechanical load previously studied, the thermal expansion does not "break" the in-plane isotropy, thus the shear stresses and strains are null and the mechanical states independent of the orientation angle $\Theta$ of the considered reinforcing strip. One would notice that this result is also valid for any load respecting the in-plane symmetry.

The local stresses inside the in-plane isotropic material are indicated in the Table 8 below. At the intermediate scale, one can observe a marked gap between the $\sigma_{x x}$ stresses in the two constituents: the matrix is subjected to

Table 6

Averages of the localization and concentration tensors for the in-plane isotropic material, with the mixed formulation.

\begin{tabular}{lllllll}
\hline$\left\langle A^{i}\right\rangle$ & $A_{11}=A_{22}$ & $A_{33}$ & $A_{44}=A_{55}$ & $A_{66}$ & $A_{12}=A_{21}$ & $A_{13}=A_{23}$ \\
\hline & 1.029 & 1.000 & 0.500 & 0.511 & 0.007 & $A_{32}$ \\
$\left\langle B^{i}\right\rangle$ & $B_{11}=B_{22}$ & $B_{33}$ & $B_{44}=B_{55}$ & $B_{66}$ & $B_{12}=B_{21}$ & $B_{13}=B_{23}$ \\
\hline & 1.001 & 1.001 & 0.497 & 0.500 & 0.001 & -0.009 \\
Expected value & 1 & 1 & $1 / 2$ & $1 / 2$ & 0.001 & 0 \\
\hline
\end{tabular}

Table 7

CTE of the in-plane isotropic material estimated by the self-consistent model, for the mixed formulation, and associated errors.

\begin{tabular}{|c|c|c|c|c|c|c|}
\hline & \multicolumn{2}{|l|}{ CTE } & \multicolumn{2}{|c|}{ Error on $\varepsilon$} & \multicolumn{2}{|c|}{ Error on $\sigma$} \\
\hline & $\alpha_{1}, \alpha_{2}\left(10^{-6} / \mathrm{K}\right)$ & $\alpha_{3}\left(10^{-6} / \mathrm{K}\right)$ & $X_{1}^{\text {thermo }}$ & $X_{3}^{\text {thermo }}$ & $Y_{1}^{\text {thermo }}$ & $Y_{3}^{\text {thermo }}$ \\
\hline$H$ mixed & 3.52 & 64.3 & 0.031 & 0.027 & 0.004 & 0.002 \\
\hline Expected value & $\sim 4$ & $\sim 60$ & 0 & 0 & 0 & 0 \\
\hline
\end{tabular}



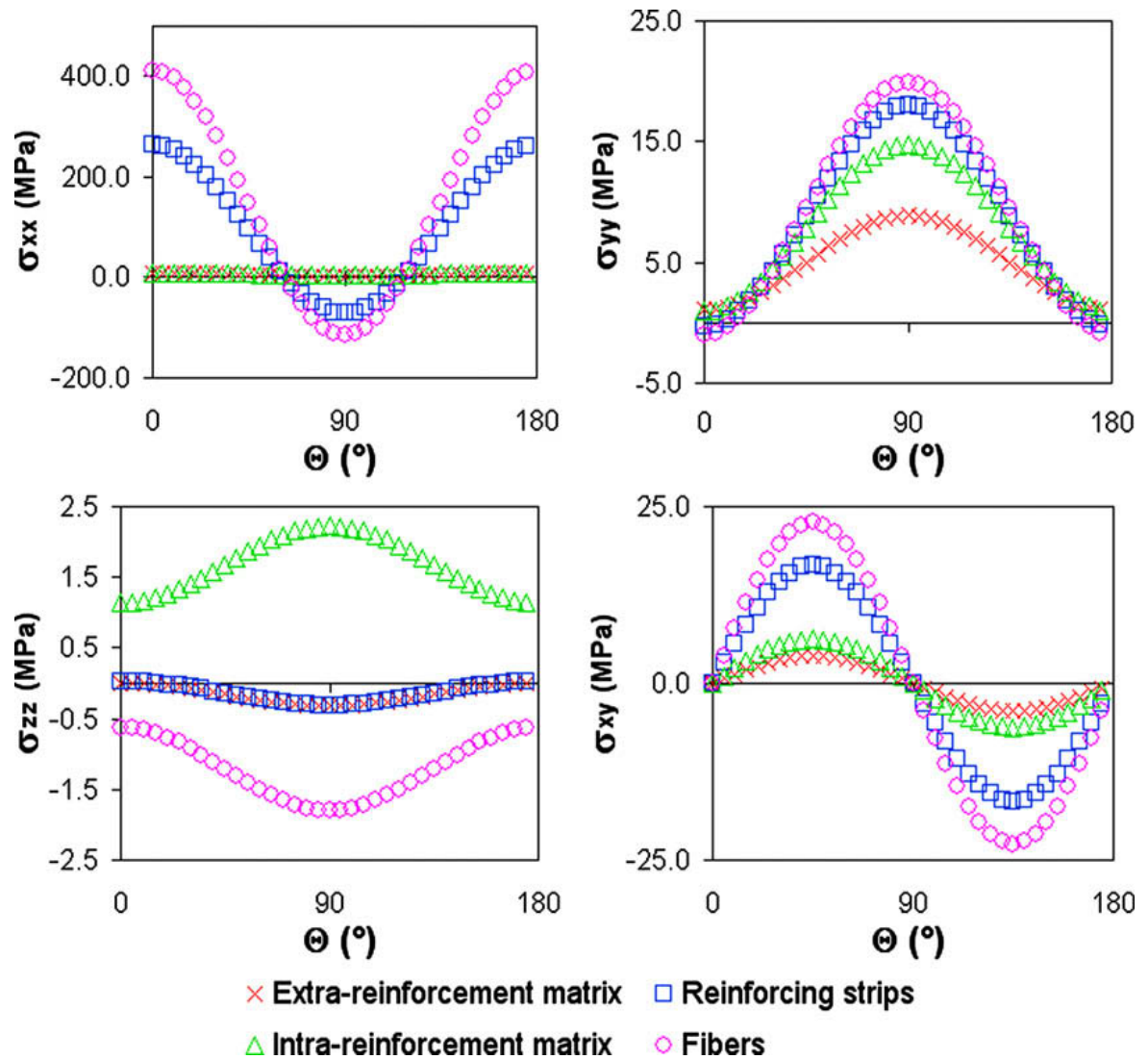

Fig. 3. Local stress states in the constituents of the material submitted to a $100 \mathrm{MPa}$ mechanical load.

Table 8

Stress states in the material and its constituents under a $-100{ }^{\circ} \mathrm{C}$ thermal load.

\begin{tabular}{llrrr}
\hline Scale & Medium & \multicolumn{3}{c}{ Stresses (MPa) } \\
\cline { 3 - 5 } & & \multicolumn{1}{c}{$\sigma_{x x}$} & \multicolumn{1}{c}{$\sigma_{y y}$} & \multicolumn{1}{c}{$\sigma_{z z}$} \\
\hline Macroscopic & Effective material & 0.0 & 0.0 & 0.0 \\
Intermediate & Extra-reinforcement & 42.6 & 42.5 & 0.4 \\
& matrix & & & \\
& Reinforcing strips & -48.1 & 44.1 & 0.1 \\
Microscopic & Intra-reinforcement & 57.79 & 55.51 & 25.39 \\
& matrix & -109.9 & 37.5 & -14.7 \\
\hline
\end{tabular}

traction, although the reinforcing strips are constricted; besides, this compression state implies a risk of microbuckling at the surface of the material. The same scheme appears at the microscopic scale, where the fibers are compressed and the matrix stretched. Along the normal direction $z$, one can also notice the emergence of complementary stresses in the fibers and the matrix, due to the gap on properties between these two constituents.

\section{Discussion and prospectives}

A two-steps scale transition procedure based on Eshelby's inclusion has been introduced in order to describe the thermo-mechanical behaviour of an in-plane isotropic composite material, made of epoxy resin and carbon-epoxy reinforcing strips, exhibiting an in-plane distribution on the morphologies. The limits of the self-consistent model for this kind of microstructures have been discussed, by comparing the results of the strain-based and stress-based formulations; each solution was discussed on the base of Hill's averages principles. Referring to these as validity criterions, a mixed formulation was then suggested and gave satisfying results for thermo-mechanical properties and mechanical states averages.

The previous framework and its results are applicable to other particles-reinforced materials such as nanocomposites, as the morphologies tested are close to those exhibited by carbon nanotubes. Thereby, the multimorphous model is an interesting alternative to the Krenchel model (Krenchel, 1964; Thostenson and Chou, 2003) which gives a simple estimate of the elastic properties of isotropic particles-reinforced materials.

The scale transition procedure was applied to two typical thermo-mechanical loadings. This enabled to calculate the local stresses in the material when experiencing mechanical and thermal loads. Theses loads and stresses are typical of those undergone by carbon-epoxy composite materials during the cooling of cure processes, and constitute an important issue for industrial applications. The complex evolution of residual stresses during cure process is mainly induced by the reticulation reaction of the organic matrix, with the associated chemical shrinkage, and the evolution of its properties. These stresses can be predicted within the above developed framework.

To conclude, this work has shown that the multi-morphous self-consistent model is a powerful tool for the 
prediction of homogenized properties of composite materials and residual (or service) local stress states. However, for HEMs constituted by inclusions with very distinct morphologies, special attention must be paid to the uniqueness of the solution and the verification of Hill's averages principles.

\section{References}

Agbossou, A., Pastor, J., 1997. Thermal stresses and thermal expansion coefficients of n-layered fiber-reinforced composites. Composite Science and Technology 57, 249-260.

Baptiste, D., 2003. Non linear behavior micromechanical multi-scale modelling of discontinuous reinforced composites. Materials Science Forum, 3939-3944.

Benveniste, Y., 1987. A new approach to the application of Mori-Tanaka's theory in composite materials. Mechanics of Materials 6, 147-157.

Benveniste, Y., Dvorak, G.J., Chen, T., 1991. On diagonal and elastic symmetry of the approximate effective stiffness tensor of heterogeneous media. Journal of the Mechanics and Physics of Solids 39, 927-946.

Berryman, J., Berge, P., 1996. Critique of two explicit schemes for estimating elastic properties of multiphase composites. Mechanics of Materials 22, 149-164.

Chen, T., Dvorak, G.J., Benveniste, Y., 1992. Mori-Tanaka estimates of the overall elastic moduli of certain composite materials. Journal of applied mechanics 59:3, 539-546.

Eshelby, J.D., 1957. The Determination of the Elastic Field of an Ellipsoidal Inclusion, and Related Problems. Proceedings of the Royal Society London A241, 376-396.

François, M., 1991. Détermination des contraintes résiduelles sur des fils d'acier euctectoïde de faible diamètre par diffraction des rayons X, Doctoral Thesis, ENSAM Paris.

Fréour, S., 2003. Application des modèles de transition d'échelle à la détermination des contraintes internes par diffraction des rayons $\mathrm{X}$, cas des matériaux biphasés, Doctoral Thesis, University of Nantes.

Fréour, S., Jacquemin, F., Guillén, R., 2005. On an analytical self-consistent model for internal stress prediction in fiber-reinforced composites submitted to hygroelastic load. Journal of Reinforced Plastics and Composites 24, 1365-1377.

Fréour, S., Jacquemin, F., Guillén, R., 2006. Extension of Mori-Tanaka approach to hygroelastic loading of fiber-reinforced Composites comparison with Eshelby-Kröner self-consistent model. Journal of Reinforced Plastics and Composites 25, 1039-1053.

Guemes, J.A., 1994. Curing residual stresses and failure analysis in composite cylinders. Journal of Reinforced Plastics and Composites 13, 408-419.

Hill, R., 1965. Continuum micro-mechanics of elastic polycrystals. Journal of the Mechanics and Physics of Solids 13, 89-101.
Hill, R., 1967. The essential structure of constitutive laws for metals composites and polycrystals. Journal of the Mechanics and Physics of Solids $15,79-95$.

Hutchinson, J.W., 1970. Elastic-plastic behaviour of polycrystalline metals and composites. Proceedings of the Royal Society London 319, 247-272.

Jacquemin, F., Fréour, S., Guillén, R., 2005. A hygroelastic self-consistent model for fiber-reinforced composites. Journal of Reinforced Plastics and Composites 24, 485-502.

Kocks, U.F., Tomé, C.N., Wenk, H.R., 1998. Texture and Anisotropy. Cambridge University Press.

Krenchel, H., 1964. Fibre Reinforcements. Akademisk Forlag, Copenhagen.

Kröner, E., 1958. Berechnung der elastischen Konstanten des Vielkristalls aus des Konstanten des Einkristalls. Zeitschrift für Physik 151, 504508.

Kröner, E., 1961. Zur plastischen verformung des vielkristalls. Acta Metallurgica 9, 155-161.

Le Pen, E., Baptiste, D., 2002. Multi-scale fatigue behaviour modelling of $\mathrm{Al} / \mathrm{Al}_{2} \mathrm{O}_{3}$ short fibre composites by a micro-macro approach. International Journal of Fatigue 24, 205-214.

Morris, R., 1970. Elastic constants of polycrystals. International Journal of Engineering Science 8 (49), 49-61.

Mura, T., 1982. Micromechanics of Defects in Solids. Martinus Nijhoff Publishers, The Hague, Netherlands.

Ogi, K., Kim, H.S., Maruyama, T., Takao, Y., 1999. The influence of hygrothermal conditions on the damage processes in quasi-isotropic carbon/epoxy laminates. Composites Science and Technology 59, 2375-2382.

Pham, D.C., 2000. Weighted self-consistent approximations for elastic completely random mixtures. Mechanics of Materials 32, 463-470.

Qiu, Y.P., Weng, G.J., 1991. The influence of inclusion shape on the overal elastoplastic behavior of a two-phase isotropic composite International Journal of Solids and Structures 27 (12), 1537-1550.

Roe, R.J., 1965. Description of crystallite orientation in polycrystalline materials, III. General solution to pole figure inversion. Journal of Applied Physics 36, 2024-2031.

Thostenson, ET, Chou, T.W, 2003. On the elastic properties of carbon nanotube-based composites: modeling and characterization. Journal of Physics Part D: Applied Physics 36, 573-582.

Treacy, M.M.J., Ebbesen, T.W., Gibson, T.M., 1996. Exceptionally high Young's modulus observed for individual carbon nanotubes. Nature 381, 678-680.

Vook, R.W., Witt, F., 1968. Thermally induced strains in cubic metal films. Journal of Applied Physics 39, 2773-2776.

Welzel, U., Fréour, S., 2007. Extension of the Vook-Witt and inverse Vook-Witt elastic grain interaction models to general loading states. Philosophical Magazine 87, 3921-3943.

Weng, G.J., 1993. A self-consistent relation for the time-dependent creep of polycrystals. International Journal of Plasticity 9, 181-198. 